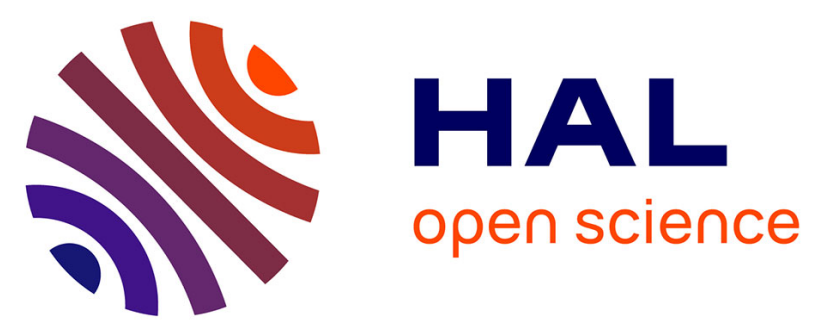

\title{
Numerical investigation of the relative effect of disc bulging and ligamentum flavum hypertrophy on the mechanism of central cord syndrome
}

Nicolas Bailly, Lucien Diotalevi, Marie-Hélène Beausejour, Eric Warnac, Jean-Marc Mac-Thiong, Yvan Petit

\section{To cite this version:}

Nicolas Bailly, Lucien Diotalevi, Marie-Hélène Beausejour, Eric Warnac, Jean-Marc Mac-Thiong, et al.. Numerical investigation of the relative effect of disc bulging and ligamentum flavum hypertrophy on the mechanism of central cord syndrome. Clinical Biomechanics, 2020, 74, pp.58-65. 10.1016/j.clinbiomech.2020.02.008 . hal-03226854

\section{HAL Id: hal-03226854 \\ https://hal-amu.archives-ouvertes.fr/hal-03226854}

Submitted on 15 May 2021

HAL is a multi-disciplinary open access archive for the deposit and dissemination of scientific research documents, whether they are published or not. The documents may come from teaching and research institutions in France or abroad, or from public or private research centers.
L'archive ouverte pluridisciplinaire HAL, est destinée au dépôt et à la diffusion de documents scientifiques de niveau recherche, publiés ou non, émanant des établissements d'enseignement et de recherche français ou étrangers, des laboratoires publics ou privés. 
Bailly, Nicolas ${ }^{a, b, c}$; Diotalevi, Lucien ${ }^{a, b, c}$; Beausejour Marie-Helene ${ }^{a, b, c, d}$, Wagnac, Éric ${ }^{a, b, c}$; MacThiong, Jean-Marc ${ }^{b, e} ;$ Petit, Yvan ${ }^{a, b, c}$

${ }^{a}$ Department of Mechanical Engineering, École de technologie supérieure 1100 Notre-Dame Street West

Montréal, Québec, H3C 1K3, Canada

${ }^{\text {b }}$ Research Center, Hôpital du Sacré-Cœur de Montréal 5400 Gouin blvd Montréal, H4J 1C5, Québec, Canada

${ }^{\mathrm{C}}$ International Laboratory on Spine Imaging and Biomechanics (iLab-Spine)

${ }^{d}$ Laboratoire de Biomécanique Appliquée

UMRT24 IFSTTAR- Université de la Méditerranée

F-13916 Marseille cedex 20, France

${ }^{\text {e }}$ Department of Orthopaedic Surgery, Université de Montréal

P.O. box 6128, Station Centre-Ville

Montréal, Québec, H3C 3J7, Canada

\section{Corresponding author:}

Yvan Petit, P.Eng, Ph.D.

Professor

École de technologie supérieure

Department of Mechanical Engineering

1100 Notre-Dame Street, West

Montréal, Québec, H3C 1K3, Canada

E-mail: $\quad$ yvan.petit@etsmtl.ca

\section{Word count}

Abstract: 235

Main text: 3855 


\section{Abstract}

45 Background: The pathogenesis of the central cord syndrome is still unclear. While there is a 46 consensus on hyperextension as the main traumatic mechanism leading to this condition, there

47 is yet to be consensus in studies regarding the pathological features of the spine (intervertebral 48 disc bulging or ligamentum flavum hypertrophy) that could contribute to clinical manifestations.

49 Methods: A comprehensive finite element model of the cervical spine segment and spinal cord 50 was used to simulate high-speed hyperextension. Four stenotic cases were modelled to study 51 the effect of ligamentum flavum hypertrophy and intervertebral disc bulging on the von Mises 52 stress and strain.

53 Findings: During hyperextension, the downward displacement of the ligamentum flavum and a 54 reduction of the spinal canal diameter (up to 17\%) led to a dynamic compression of the cord. 55 Ligamentum flavum hypertrophy was associated with stress and strain (peak of $0.011 \mathrm{Mpa}$ and 560.24 , respectively) in the lateral corticospinal tracts, which is consistent with the histologic 57 pattern of the central cord syndrome. Linear intervertebral disc bulging alone led to a higher 58 stress in the anterior and posterior funiculi (peak $0.029 \mathrm{Mpa}$ ). Combined with hypertrophic 59 ligamentum flavum, it further increased the stress and strain in the corticospinal tracts and in 60 the posterior horn (peak of $0.023 \mathrm{Mpa}$ and 0.35, respectively).

61 Interpretation: The stenotic typology and geometry greatly influence stress and strain 62 distribution resulting from hyperextension. Ligamentum flavum hypertrophy is a main feature 63 leading to central cord syndrome.

\section{Keywords}

65 Central cord syndrome, hyperextension, cervical spine, spinal cord, spondylosis 


\section{Introduction}

70 Central cord syndrome (CCS) is the most common type of incomplete traumatic cervical spinal 71 cord injury and its incidence is increasing in older patients (McKinley et al., 2007; Thompson et 72 al., 2015). This syndrome is characterized by a greater motor impairment in the upper limbs 73 than in the lower limbs. It was first described in 1954 by Schneider et al. (Schneider et al., 1954), 74 and was attributed to hemorrhage damages and swelling, predominantly in the spinal grey 75 matter and in the lateral corticospinal tracts. Later, MRI and histopathological studies 76 substantiated this, showing a predominance of axonal injury in the lateral column (Collignon et 77 al., 2002; Jimenez et al., 2000; Martin et al., 1992; Quencer et al., 1992).

78 The pathogenesis of CCS was initially described as a compression of the spinal cord during hyperextension of the neck, usually combined with stenosis of the cervical spinal canal (Molliqaj et al., 2014; Schneider et al., 1954). However, the main stenotic features of the spine contributing to CCS are still debated: the spinal cord is either compressed posteriorly from anterior bulging of the ligamentum flavum (Schneider et al., 1954), anteriorly from marginal osteophytes, anteriorly from disc bulging (Dai et al. 2000), or by any combination of those three features. Histological and MRI studies suggested that axonal injury was mainly located in the lateral columns (Collignon et al., 2002; Jimenez et al., 2000; Martin et al., 1992; Quencer et al., 1992), which may then be the location of maximal stresses in the spinal cord in the presence of CCS. Unfortunately, histological studies only present indirect evidence from a posteriori evaluations, with little understanding of the mechanism of injury.

The latest advances in finite element (FE) analysis enable the replication of such injury mechanisms and the estimation of the strain and stress distribution in the spinal cord throughout traumatic events. Li et al. (2010) simulated the extension, flexion and compression of the cervical spinal cord submitted to an anterior or posterior compressive force. Nishida et al. (2012) simulated extension with the cervical spinal cord submitted to posterior compression from a simplified hypertrophic ligamentum flavum model. These studies showcased peak stresses located in the grey matter and both anterior and posterior funiculi, as opposed to histopathological studies reporting the predominance of axonal injury in the lateral column

97 (Martin et al., 1992; Quencer et al., 1992). Therefore, the exact pathogenesis of traumatic central cord syndrome remains largely debated, although it affects an increasing number of patients (Thompson et al., 2015). 
The goal of this paper is to investigate the injury mechanisms of traumatic CCS. Using a comprehensive cervical FE model, this study will specifically assess the relative contributions of intervertebral disc bulging and ligamentum flavum hypertrophy on the stress and strain distributions within the spinal cord during an hyperextension of the neck.

\section{Methods}

\subsection{Finite element modelling of the cervical spine}

A finite element (FE) model of the cervical spine (C2-T1) described in previous works (Beauséjour et al., 2019; Fradet et al., 2014; Taso et al., 2015) was adapted for this study. This model of a $50^{\text {th }}$ percentile adult male subject includes vertebrae, intervertebral discs, as well as six spinal ligaments: anterior and posterior longitudinal ligaments, zygapophyseal joint ligaments, ligamentum flavum, interspinous ligaments and nuchal ligament (Fig. 1(a)).

(a) Finite element model of the cervical spine

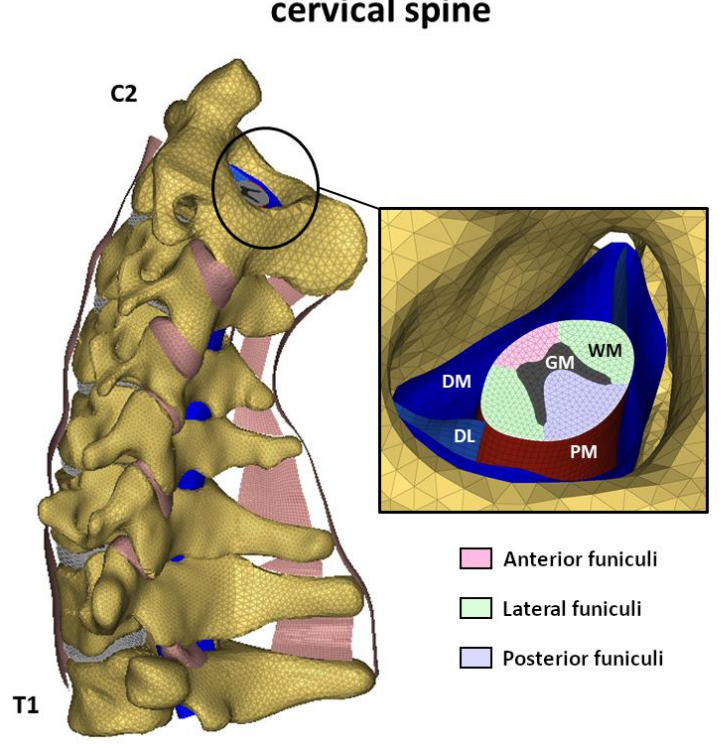

\section{(b) Stenotic scenarios at C5-C6 level}

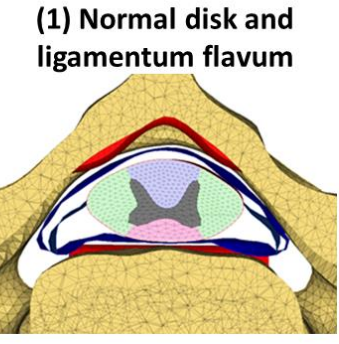

(3) Hypertrophic ligamentum flavum

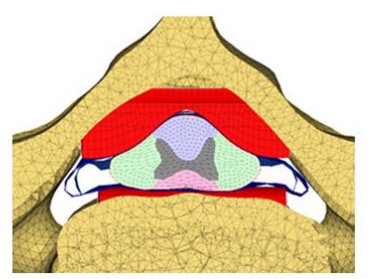

(2) Disk bulging

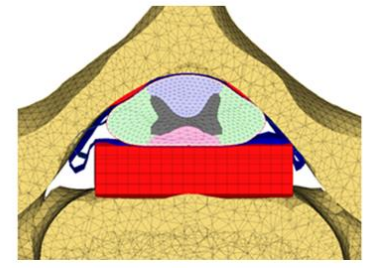

(4) Disk bulging and Hypertrophic ligamentum flavum

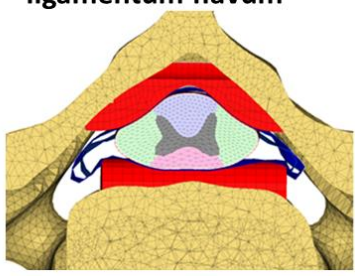

Fig. 1. (a) Finite element model of the cervical spine and (b) Stenotic scenario at C5-C6 levels in

Each vertebra was defined as a rigid body. Material laws for the ligaments were defined by tabulated, non-linear, strain-rate dependent, stress-strain curves derived from mean forcedeflection curves measured in experimental studies (Mattucci et al., 2012; Mattucci and Cronin, 2015). The interspinous ligament force-deflection curve was applied to the adjacent nuchal 
119 ligament, as this ligament was not included in the experimental studies. Toe regions of the 120 curves were calibrated in a previous study to account for the initial elongation of cervical 121 ligaments (Beauséjour et al., 2019). The intervertebral discs were divided into a nucleus 122 pulposus ( $20 \%$ of the disk volume) and an annulus fibrosus ground substance. They were then 123 associated with first-order Mooney-Rivlin hyper-elastic material laws. The annulus fibrosus was 124 divided into three sections (anterior, posterior and lateral) reinforced with specific tension-only 125 spring elements organized in concentric lamellae with a crosswise pattern at $\pm 35^{\circ}$ to represent 126 the collagen fibers (Wagnac et al., 2011). The initial material properties of the discs were initially 127 defined from Schmidt et al. (2006) and Shirazi-adl et al. (1986), and then modified and calibrated

128 for dynamic flexion and extension, as described in section 2.2.

129 The FE model also includes a spinal cord model previously described by Fradet et al. (2014), Taso 130 et al. (2015) and Diotalevi et al. (2020). It is composed of white and grey matter, denticulate 131 ligaments, pia mater and dura mater (Fig. 1 (a)). Meshing was performed with pentahedral (6 132 nodes) 3D elements for the white and grey matter, and quadratic (4 nodes) shell elements for

133 the ligaments, the pia and the dura mater. The proximal end of the spinal cord was attached to 134 the C2 vertebra to simulate the connection with the brain stem and to guide the spinal cord 135 movement. The rest of the spinal cord was left unrestrained. Material laws and properties of the 136 FE model are summarized in Table 1.

Table 1: Finite element model material properties

\begin{tabular}{|c|c|c|c|c|}
\hline & $\begin{array}{l}\text { Type of } \\
\text { elements }\end{array}$ & Material law & $\begin{array}{l}\text { Material parameters } \\
\text { (units: } \mathrm{g}, \mathrm{mm}, \mathrm{ms} \text { ) }\end{array}$ & References \\
\hline \multicolumn{5}{|l|}{ Spinal cord: } \\
\hline Grey matter & Pentahedral Brick & Stress-strain tabulated & $\begin{array}{l}\text { Curves in Fradet et al. } \\
2016\end{array}$ & (Fradet et al., 2016) \\
\hline White matter & Pentahedral Brick & Stress-strain tabulated & $\begin{array}{l}\text { Curves in Fradet et al. } \\
2016\end{array}$ & (Fradet et al., 2016) \\
\hline Pia mater & Quad & Linear elastic & $\rho=0.001 ; v=0.45 ; E=2.3$ & (Fradet et al., 2016) \\
\hline Dura mater & Quad & Linear elastic & $\rho=0.001 ; v=0.45 ; E=5$ & (Fradet et al., 2016) \\
\hline Dentate ligament & Quad & Linear elastic & $\rho=0.001 ; v=0.45 ; E=10$ & (Fradet et al., 2016) \\
\hline \multicolumn{5}{|l|}{ Ligaments: } \\
\hline & $\begin{array}{l}\text { Quad } \\
\text { Except capsular } \\
\text { ligament (tria) }\end{array}$ & Stress-strain nonlinear curves & $\begin{array}{l}\text { Curves derived from } \\
\text { Mattucci and Cronin } 2015\end{array}$ & $\begin{array}{ll}\text { (Mattucci } & \text { and } \\
\text { Cronin, 2015) } & \end{array}$ \\
\hline
\end{tabular}


140 The dynamic calibration of the model was performed by adjusting the material properties of the 141 annulus extracellular matrix and nucleus until the moment-rotation curves of each functional 142 spinal unit (C2-C3 to C7-T1) fit with experimental results from Barker et al. ( 2014). For each 143 spinal unit, the lower vertebra was fixed, while a rotation of 500 deg. $\mathrm{s}^{-1}$ in flexion and extension 144 was applied to the upper vertebra. The axis of rotation of each unit was determined as 145 described in Amevo et al. (1991) to replicate the experimental conditions of Barker et al. (2014). 146 Since the C7-T1 axis was not included in this study, the data for C6-C7 was used to set the axis of 147 rotation.

148 To determine the goodness of fit between simulation and experimental results, three criteria 149 were used: corridor rating, shape rating and size rating. For each data point, the corridor rating 150 was set to 1 if the simulation results fit inside one standard deviation, and to 0 if the result was 151 outside two standard deviations. For results between one and two standard deviations, a linear 152 relation between 0 (outside 2 standard deviations) and 1 (equal to one standard deviation) was 153 calculated. The final corridor rating value was obtained by averaging corridor rating values at 154 every data point. The size rating and shape rating were measured as defined in Barker et al. 155 (2017). The size rating (equation (1)) is the ratio between the area below the simulation curve $156 \mathrm{y}(\mathrm{t})$ and the experimental curve $\mathrm{x}(\mathrm{t})$. The larger value is set as the denominator.

$$
\text { Size rating }=\frac{\sum_{i=1}^{n} y^{2}\left(t_{\min }+i * \Delta t\right)}{\sum_{i=1}^{n} x^{2}\left(t_{\min }+(m+i) * \Delta t\right)}
$$

The shape rating (equation (2)) compares the slope and the change in the slope of the simulated curve $y(t)$ against the experimental curve $x(t)$. The phase-shifting $m$, representing the $x$-intercept shifting, was not enabled for the measure of the shape rating. Therefore, $m$ was set to 0 .

$$
\text { Shape rating }=\frac{\sum_{i=0}^{n-1} x\left(t_{\text {min }}+(m+i) * \Delta t\right) * y\left(t_{\text {min }}+i * \Delta t\right)}{\sqrt{\sum_{i=0}^{n-1} x^{2}\left(t_{\min }+(m+i) * \Delta t\right) * \sum_{i=0}^{n-1} y^{2}\left(t_{\min }+i * \Delta t\right)}}
$$

An average of these three criteria was calculated for the moment-rotation curves in flexion and extension, and an overall rating was measured as the average between the rate in flexion and extension. It was chosen to target a rating of between 0.65 and 1 , based on a rating scale defined by Cesari et al. (2001). 
Calibration of the disk material properties was performed by adjusting the parameters $\mathrm{C}_{10}$ and

$167 \mathrm{C}_{01}$ of the Mooney-Rivlin hyper-elastic material laws used for the annulus and nucleus for each spinal unit. First, the dynamic flexion and extension of each unit were performed with the quasistatic material properties of the disk (Schmidt et al., 2006). If the ratings met our criteria, no calibration was performed. Otherwise, a scaling factor of 0.5 to 15 was applied to the annulus and nucleus rigidity (parameters $C_{10}$ and $C_{01}$ of the material law), while maintaining the $C_{10}$ to $C_{01}$ ratio. The range of 0.5 to 15 was chosen to vary the properties grossly between values 173 determined in other studies for the lumbar disk in quasi-static (Schmidt et al., 2006) and 174 dynamic (Wagnac et al., 2012) loadings. A $C_{10}$-to- $C_{01}$ ratio was maintained based on the results 175 of a preliminary sensitivity study, which showed that this ratio had no significant impact on the 176 FSU mobility. If no satisfying result was obtained, the rigidity of the nucleus and the anterior, 177 lateral and posterior bulk annuli were independently iteratively scaled until the best overall rating (extension and flexion) was obtained.

\subsection{Finite element modelling of the spondylotic stenosis}

180 The original geometry of the vertebral canal was modified from C4 to C7 to simulate a stenotic canal area. First, the canal diameter was reduced by $3 \mathrm{~mm}$, corresponding to the average reduction seen in patients with spondylosis (Shedid and Benzel, 2007). Two spondylotic features were also included: linear disc bulging and hypertrophic ligamentum flavum. Four cases were modelled, based on the presence or absence of these two features (Fig. 1 (b)).

As canal stenosis associated with cervical spondylosis typically occurs at several levels, these

186 features were inserted at three intervertebral levels: C4-C5, C5-C6 and C6-C7 (displayed in red in 187 Fig. 3) (Stevenson et al., 2016). Hypertrophic ligamentum flavum and linear disk bulging 188 idealized geometries (Fig. 1 (b)) were defined based on MRI measurements of typical cases 189 found in our institution database (Thompson et al., 2015), and were consistent with the 190 literature (Muhle et al., 1998; Song et al., 2006; Tani et al., 1999; Yu et al., 1983). Idealization of 191 these features is also in accordance with the literature (Kim et al., 2013). These features were 192 meshed with hexahedral (8 nodes) 3D elements and defined as rigid bodies.

193 Modelling of the ligamentum flavum hypertrophy and disc bulging was performed in a 194 preliminary simulation, with the neck placed in an upright position, and a normal C2-C7 lordosis 195 angle of $22.7^{\circ}$ according to Gore et al. (1986). The spondylotic features were moved alongside 
196 the anteroposterior axis into the canal at the three affected levels to compress the cord and

197 reach $40 \%$ reduction of the sagittal canal diameter, which corresponds to a typical degree of 198 compression in cervical spondylosis, according to Ogino et al. (1983). Then, the disk bulging 199 features were fixed to the lower vertebra while the hypertrophic ligamentum flavum features 200 were fixed to the upper vertebra of each segment. These altered geometries were then used in 201 the neck hyperextension simulation. Both spondylotic features had frictionless contacts with the 202 dura mater.

\section{2.4. Loading and boundary conditions}

204 Hyperextension was achieved by fixing T1 in space and applying 60 degrees of rotation in the 205 median plane to $\mathrm{C} 2$ at $500 \mathrm{deg} \cdot \mathrm{s}^{-1}$, around a mobile axis located at the center of the vertebral 206 foramen of $\mathrm{C} 2$. This rotational speed of $500 \mathrm{deg} \cdot \mathrm{s}^{-1}$ has been observed during typical car crash 207 scenarios (Panzer et al., 2011) and a $60^{\circ}$ magnitude of rotation approximates the maximal 208 amplitude, without soft-tissue injury, as reported by Barker et al. (2014).

\section{$210 \quad 2.5$ Analysis}

211 Model outcomes were von Mises strains and stresses in the grey and white matter. Both 212 outcomes were extracted at every node of the FE model. A simple averaging of their values was 213 performed by summing the studied tensor equivalent invariant measured the finite element 214 corner adjacent to the studied node, and then dividing them by the number of finite element 215 corners adjacent to this node. This method was used to avoid mathematical aberrations on the 216 results.

\section{3. Results}

\section{3.1. Dynamic calibration}

219 Goodness of fit scores between the model's FSU kinematics and experimental results from 220 Barker et al. (2014) varied from 0.82 to 0.90 (Fig. 2). The resulting $C_{01}$ and $C_{10}$ parameters for

221 the annulus and nucleus are presented in Appendix 1. The moment-rotation curves generally lie 222 within one standard deviation of the experimental results (overall corridor ratings between 0.76 223 and 1) (Fig. 2). 

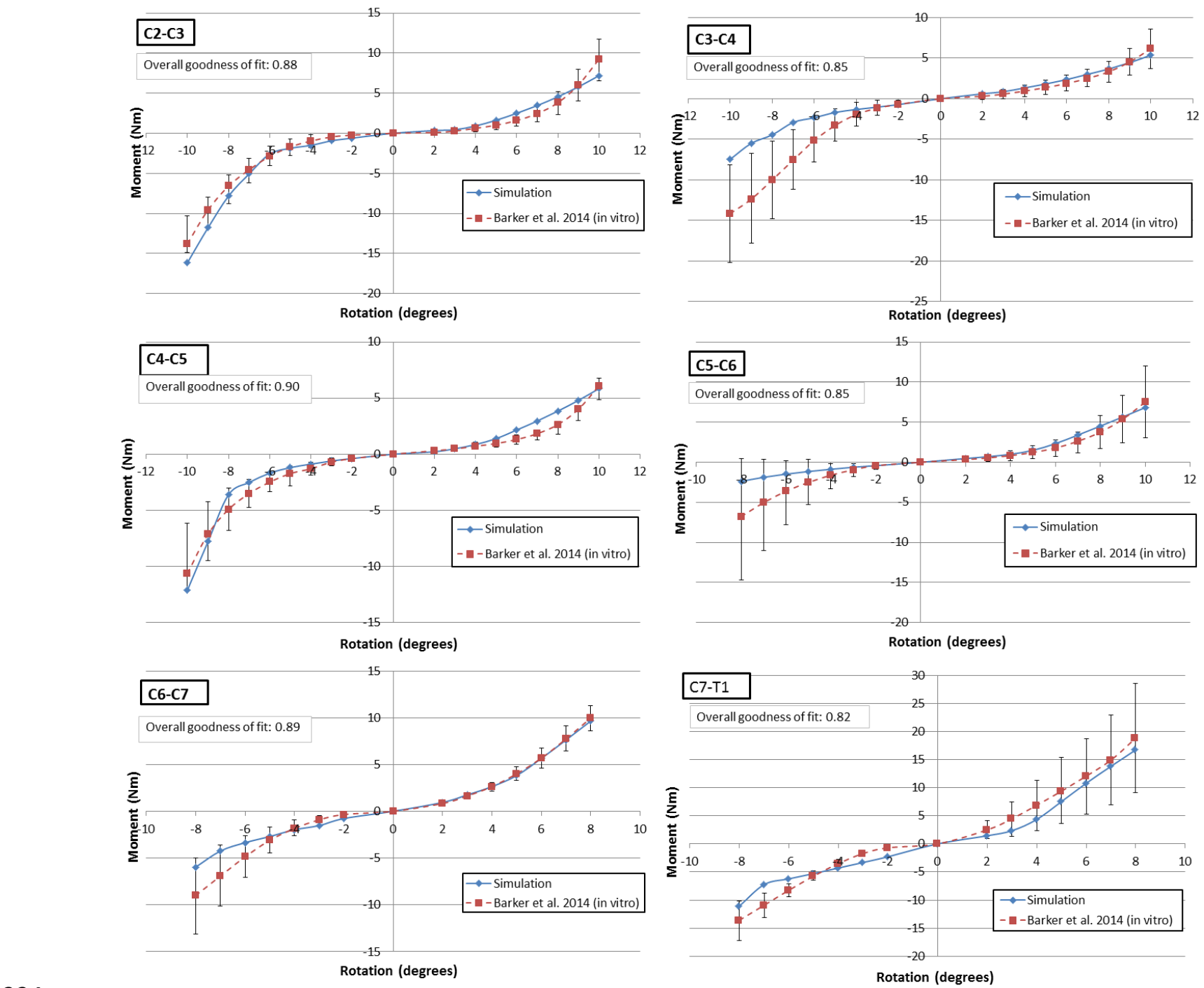

Fig. 2: Cervical FSU moment-rotation diagrams at rotational velocity of $500 \mathrm{deg} \cdot \mathrm{s}^{-1}$

\subsection{Stress and strain distribution during hyperextension}

227 Figure 3 presents a sagittal cross-section of the cervical spine during hyperextension and the 228 strain distribution in the cord. During hyperextension, the rotation of the upper adjacent 229 vertebra induced a downward displacement of the ligamentum flavum: at the C5-C6 level, the 230 ligamentum flavum, initially located above the disk, ended up below it at the end of 231 hyperextension (Fig. 3). This was associated with a reduction of the minimal spinal canal antero232 posterior diameter (up to $17 \%$ decrease at C5-C6). The location of this canal reduction also 233 shifted downward during the hyperextension: it is above the disk at $30^{\circ}$, but below the disc at $23460^{\circ}$ of extension. The highest stress and strain were observed at C5-C6 (Fig. 3). 


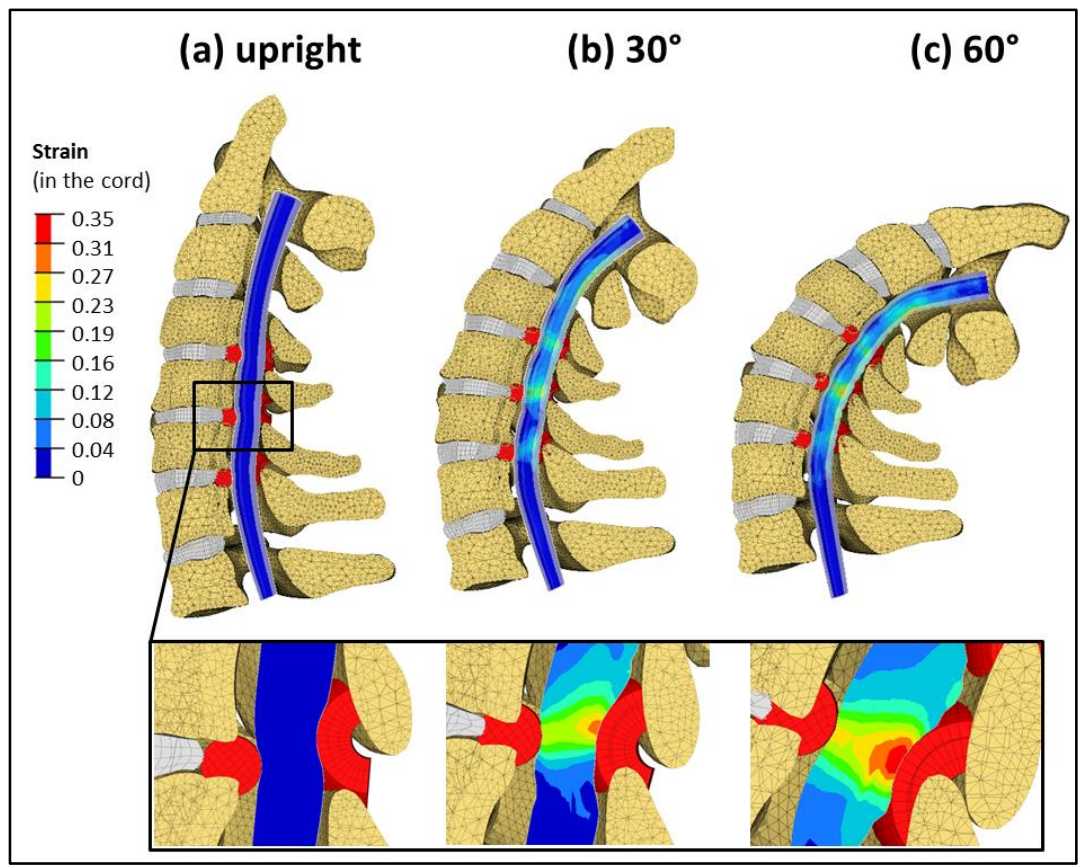

Fig. 3. Strain distribution in a sagittal cross-section of the spinal cord during hyperextension with a stenotic case presenting a narrowed canal, hypertrophic ligamentum flavum and linear

239 In the transverse plane, stress and strain at C5-C6 increased gradually throughout extension, 240 with different patterns depending on the stenotic cases (Fig. 4 and 5). We observed that the 241 stress distribution did not exactly match the strain distribution due to the higher rigidity of the 242 grey matter compared to white matter. In the normal column (reference case), the peak stress 243 and strain were located in the posterior funiculi, and were respectively equal to $0.0017 \mathrm{kPa}$ and 2440.08 at $30^{\circ}$ of extension, and $0.0045 \mathrm{kPa}$ and 0.17 at $60^{\circ}$ (Fig. 4 and 5). In the presence of linear 245 disk bulging alone, stress and strain were initiated in the anterior and posterior funiculi and 246 increased gradually in both areas. At $30^{\circ}$ extension, the peak stress and strain were respectively 2479 and 4.2 times higher than in the normal column (respectively 6.5 and 3.2 times higher at $60^{\circ}$ ). 248 The peak stress was observed in the anterior horn while the peak strain was located in the 249 anterior and posterior funiculi. In the presence of hypertrophic ligament flavum alone, the peak 250 stress and strain first appeared in the posterior and medial parts of the lateral funiculi, where 251 they increased gradually, as well as in the posterior horn. At $30^{\circ}$, the peak stress and strain were 252 respectively 4.1 and 2.62 times greater than in the normal column (respectively 2.5 and 1.5 253 times higher at $60^{\circ}$ ). In the presence of both linear disk bulging and hypertrophic ligament 
flavum, stress and strain initiated in both the posterior and medial parts of the lateral funiculi and in the anterior funiculi. At $30^{\circ}$ extension, the peak stress and strain were mainly located in

256 the posterior and medial parts of the lateral funiculi and in the posterior horn, and were 257 respectively 9.4 and 3.8 times higher than in the normal column (respectively 5.2 and 2.1 times 258 higher at $60^{\circ}$ ) (Fig. 4 and 5).

In every simulation, the presence of a hypertrophic ligamentum flavum was associated with high stresses and strains in the medial part of the lateral funiculi at the early stage of the extension, which corresponds to the main damaged region in CCS (Martin et al., 1992; Quencer et al., 1992) and to the location of the corticospinal tract. The addition of a linear disk bulging into the canal

263 in the presence of hypertrophic ligamentum flavum increased the overall level of stress in these 264 areas during extension.

Normal Disk and Ligamentum Flavum

$20^{\circ}$

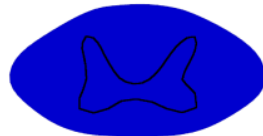

$30^{\circ}$

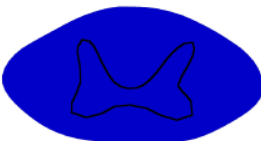

$40^{\circ}$

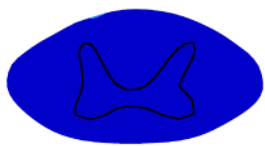

$50^{\circ}$

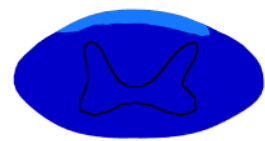

$60^{\circ}$

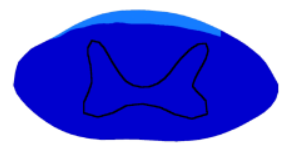

Linear Disk Bulging
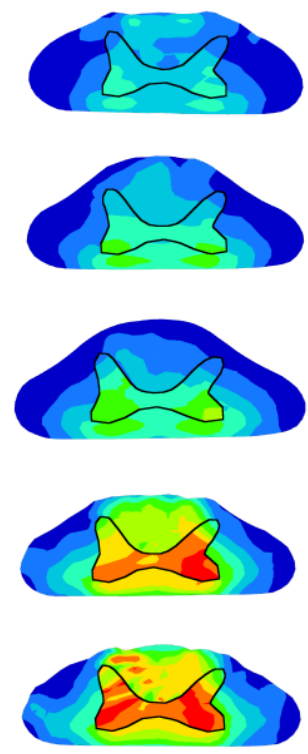

Hypertrophic Ligamentum Flavum
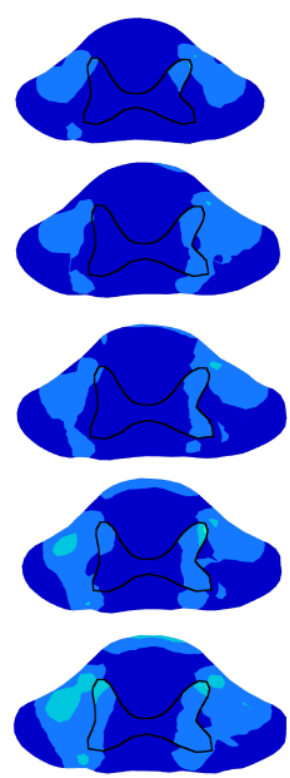

Linear Disk Bulging

\& Hypertrophic

Ligamentum Flavum

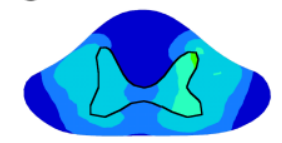

Stress

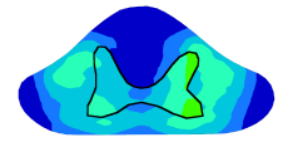

(Mpa)

$-0.03$
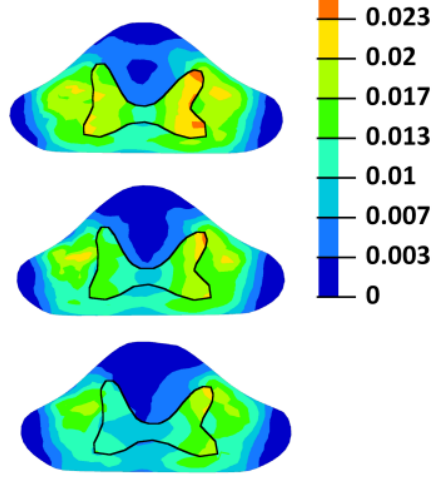
intervertebral level at 5 extension angles for the $\mathbf{3}$ different stenotic cases 


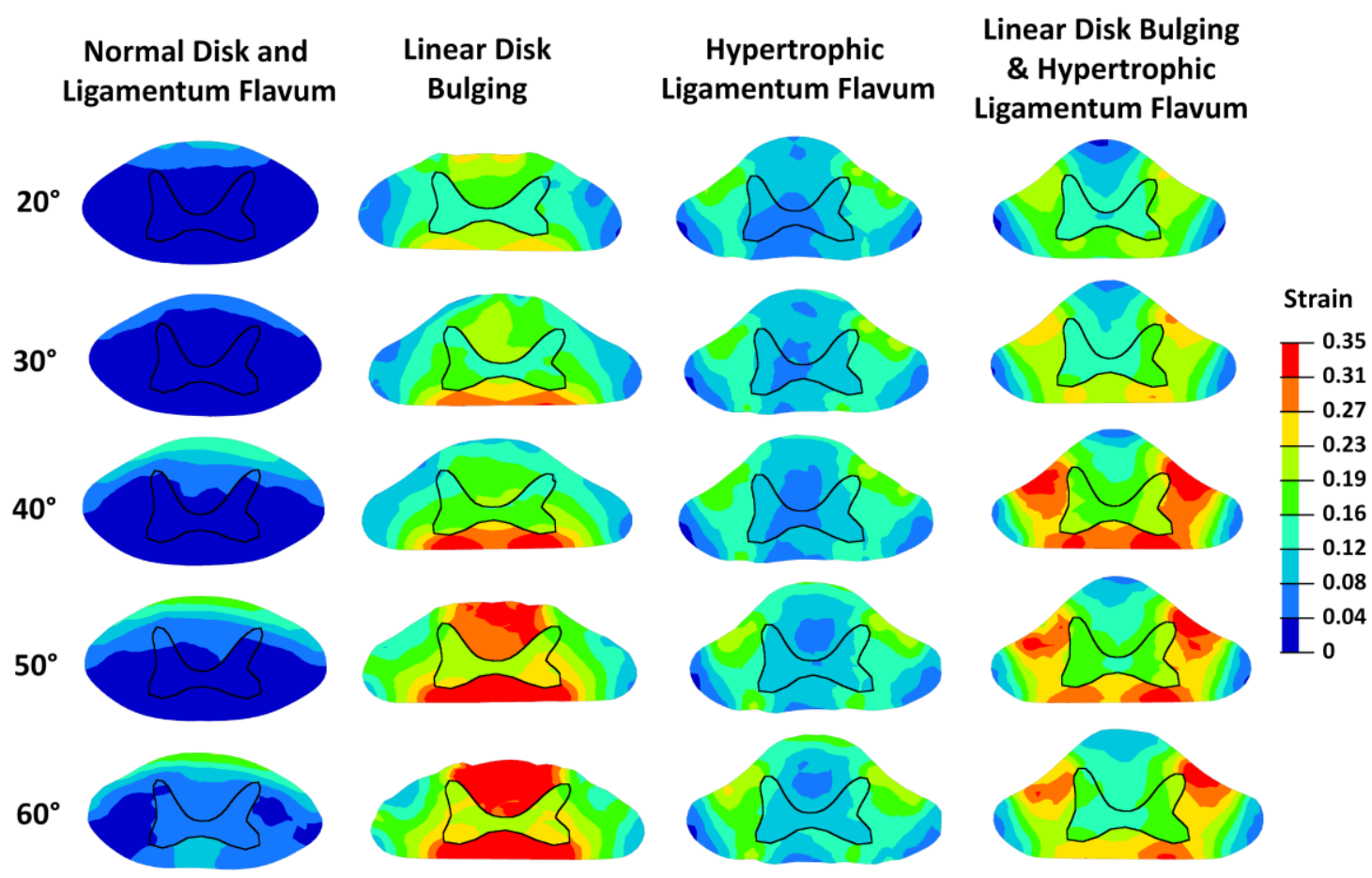

Fig. 5. Strain distributions in a transversal cross-section of the spinal cord at the C5-C6 intervertebral level at $\mathbf{5}$ extension angles for $\mathbf{3}$ different stenotic cases.

\section{Discussion}

275 This FE method study is the first to investigate the spinal cord dynamics with different cases of cervical canal stenosis leading to CCS. The results demonstrate that the geometry and position

277 of the stenotic feature greatly influence the stress and strain distributions throughout the

278 hyperextension movement of the neck. In particular, the presence of hypertrophic ligamentum 279 flavum induced specific stress and strain distributions that are consistent with histologic findings 280 related to CCS (Jimenez et al., 2000; Martin et al., 1992; Quencer et al., 1992). This provides 281 important insights into the physiopathology of CCS.

282 The mechanism of CCS is described as a transverse compression of the spinal cord, during an 283 hyperextension of the neck, due to a reduction of the cervical spinal canal size induced by the 284 bulging of the ligamentum flavum (Schneider et al., 1954). A reduction of the canal diameter 285 was indeed observed by Muhle et al. (1998) in some spondylotic patients performing a neck 286 extension in a kinematic MRI. In the present study, up to a $17 \%$ canal reduction was observed at 
the C5-C6 intervertebral level, but this was also associated with a downward displacement of 288 the ligamentum flavum (Fig. 3). This combined phenomenon modifies the pinching zone of the cord: it was above the disc in the neutral position, but below the disc at $60^{\circ}$ of extension. This might explain the significant strain increase in the cord $\left(>25 \%\right.$ at $60^{\circ}$ of extension) despite the 291 small reduction in the canal diameter ( $6 \%$ at $60^{\circ}$ of extension).

292 In the presence of the hypertrophic ligamentum flavum, the peak stress and strain were 293 observed in the posterior and medial parts of the lateral funiculi, close to the posterior horn of 294 the grey matter. This area matches those where most of the axonal damage and Wallerian 295 degeneration are found in histological studies of CCS (Jimenez et al., 2000; Martin et al., 1992; 296 Quencer et al., 1992). Conversely, stress patterns with linear disk bulging alone were mostly 297 distributed in the anterior and posterior funiculi. These results suggest that the presence of 298 hypertrophic ligament flavum is an important factor in the pathogenesis of CCS, as proposed by 299 Schneider et al. (1954) and discussed by many others (Dai and Jia, 2000; Li and Dai, 2010; 300 Quencer et al., 1992). The concomitant presence of hypertrophic ligamentum flavum and linear 301 disk bulging further increases the stresses and strains in the lateral funiculi by inducing an 302 additional pincer effect from combined anterior and posterior compression of the spinal cord 303 during extension, as suggested by previous authors (Morishita et al., 2013; Nishida et al., 2012).

304 The differences in stress and strain distributions induced by hypertrophic ligamentum flavum 305 and disk bulging may be explained by their geometry and their location of contact with the cord. In the present study, disk bulging applies a linear compression to the anterior part of the cord,

308 Conversely, the hypertrophic ligamentum flavum applies two lateral contact forces converging 309 toward the center of the spinal cord, forming a pincer movement between contralateral 310 segments of the ligamentum flavum. This induces centralized stresses and strains in the cord. In 311 previous FE method studies, Nishida et al. (2012) used other simplified geometries to model the 312 pinching force applied to the cord during hyperextension: the ligamentum flavum was modelled 313 with a linear shape, and three types of anterior compression were used (central, lateral or 314 diffuse type). Each of these configurations also presented a unique stress pattern, mainly 315 located in the grey matter and in the anterior and posterior funiculi. However, none led to high 316 levels of stress in the corticospinal tracts, as observed in the present study. This confirms that 317 different stenotic configurations lead to different stress and strain distributions, which is 

consistent with the great variability of syndromes and outcomes associated with hyperextension

319 injuries without instabilities (McKinley et al., 2007). Future FE method studies may need to 320 consider patient-specific model geometry to understand their unique injury mechanism.

321 The area of highest stress and strain concentrations generally observed in the presence of 322 hypertrophic ligamentum flavum is located in the lateral corticospinal tract, which is associated 323 with motor control, and is strongly linked with hand dexterity (Courtine et al., 2007). According 324 to Foerster (1936) and Schneider et al. (1958), the lateral corticospinal tracts have a 325 somatotopic arrangement in which the medial part is associated with upper extremity function, 326 while the lateral part is associated with lower extremity function. Based on this somatotopy, 327 greater motor impairment in the upper limbs observed in CCS would be explained by higher 328 damage in the medial part of the corticospinal tract than in the lateral part. However, this 329 hypothesis has been challenged by histologic findings showing uniform damage to the lateral 330 corticospinal tract (Quencer et al. 1992) and by Nathan and Smith (1955), who demonstrate that 331 the lateral tracts are not laminated into functional units, but rather, that the motor fiber 332 involved in upper and lower limb function were interlaced. This hypothesis was reinforced by 333 several studies on monkeys showing that sectioning of the entire corticospinal tract lead to 334 greater dysfunction in the upper limbs than in the lower limbs (Bucy et al., 1966; Nathan and 335 Smith, 1955). The results of the present study are compatible with a CCS in the two theories. On 336 the one hand, in the presence of the ligamentum flavum, high stresses were mostly distributed 337 in the corticospinal tracts, suggesting that the entire lateral corticospinal tract was affected 338 (Quencer et al. 1992; Jimenez, Marcillo, and Levi 2000). On the other hand, peak stress in the 339 white matter was mainly seen in the medial part of the lateral funiculi, next to the posterior 340 horn, which is the zone associated with upper extremity function in the somatotopic 341 arrangement described by Foerster (1936). To further correlate the stress pattern in the cord 342 with neurological damage and discriminate these two theories, future FE method studies should 343 consider reproducing patient-specific geometry and injury dynamics and compare them with 344 clinical observations.

345 This study presents some limitations associated with assumptions and simplifications. First, the 346 cerebro-spinal fluid was not included because it was absent in the stenotic zone of most cases of 347 CCS with severe spinal stenosis found in our institutional database (Thompson et al., 2015). 348 Secondly, disks and ligament behaviour in the current model were calibrated based on data 
from non-pathological spine segments (Barker et al., 2014), but a stenotic spine is expected to

350 be more rigid and less likely to sustain a $60^{\circ}$ hyperextension. However, this does not challenge 351 our conclusions since high stress and strain appeared at less than $30^{\circ}$ of extension, which is a 352 normal range of extension for the elderly, according to Muhle et al. (1998). Thirdly, the 353 mechanical behaviour of white and grey matter was defined by isotropic strain rate-dependent 354 tabulated laws derived from experiments reported in the literature (Fradet et al., 2016). 355 Nevertheless, these materials are possibly anisotropic as they are notably composed of 356 neurones, myelin, glial cells, etc. In order to further study the local stress concentrations and the 357 affected axonal tracts, future studies should be performed to supplement the understanding 358 and characterization of the local behaviour of the spinal cord white and grey matter and to 359 refine finite element models. Finally, no direct link could be established between the results of 360 this study and neurological injury because no accepted stress and strain thresholds have been 361 defined for neurological injury of the spinal cord and because the model was not associated with 362 the specific geometry and injury dynamic of a patient with identified neurological evaluation. 363 Future work should focus on reproducing patient-specific injuries and establishing a neurological 364 injury threshold.

\section{Conclusions}

This study is the first, to the authors' knowledge, to examine the dynamics of a detailed osteoligamentous spine and spinal cord model for a typical traumatic event associated with central cord syndrome. The hypothesis suggesting a pinching of the spinal cord by hypertrophic

370 ligament flavum and linear disk bulging during a hyperextension was investigated in this study. It

371 was found that only ligamentum flavum hypertrophy was associated with high stress and strain 372 in the corticospinal tracts, which is consistent with the histologic pattern of the central cord 373 syndrome. The presence of a linear disk bulging alone was not associated with such a pattern, 374 but when combined with hypertrophic ligament flavum, increased the overall level of stress and 375 strain in the corticospinal tract. 


\section{References}

Amevo, B., Worth, D., Bogduk, N., 1991. Instantaneous axes of rotation of the typical cervical motion segments: a study in normal volunteers. Clin. Biomech. 6, 111-117.

Barker, J.B., Cronin, D.S., Chandrashekar, N., 2014. High Rotation Rate Behavior of Cervical Spine Segments in Flexion and Extension. J. Biomech. Eng. 136, 121004-121004-10. https://doi.org/10.1115/1.4028107

Barker, J.B., Cronin, D.S., Nightingale, R.W., 2017. Lower cervical spine motion segment computational model validation: Kinematic and kinetic response for quasi-static and dynamic loading. J. Biomech. Eng. 139, 061009.

Beauséjour, M.-H., Petit, Y., Arnoux, P.J., Wagnac, E., 2019. Comparison of two intervertebral disc failure models in a numerical C4-C5 trauma model. Presented at the 41st Engineering in Medicine and Biology Conference., Berlin.

Bucy, P.C., Ladpli, R., Ehrlich, A., 1966. Destruction of the Pyramidal Tract in the Monkey: The Effects of Bilateral Section of the Cerebral Peduncles. J. Neurosurg. 25, 1-23. https://doi.org/10.3171/jns.1966.25.1.0001

Cesari, D., Compigne, S., Scherer, R., Xu, L., Takahashi, N., Page, M., Asakawa, K., Kostyniuk, G., Hautmann, E., Bortenschlager, K., 2001. WorldSID prototype dummy biomechanical responses. SAE Technical Paper.

Collignon, F., Martin, D., Lénelle, J., Stevenaert, A., 2002. Acute traumatic central cord syndrome: magnetic resonance imaging and clinical observations. J. Neurosurg. Spine 96, 29-33.

Courtine, G., Bunge, M.B., Fawcett, J.W., Grossman, R.G., Kaas, J.H., Lemon, R., Maier, I., Martin, J., Nudo, R.J., Ramon-Cueto, A., Rouiller, E.M., Schnell, L., Wannier, T., Schwab, M.E., Edgerton, V.R., 2007. Can experiments in nonhuman primates expedite the translation of treatments for spinal cord injury in humans? Nat. Med. 13, 561-566. https://doi.org/10.1038/nm1595

Dai, L., Jia, L., 2000. Central Cord Injury Complicating Acute Cervical Disc Herniation in Trauma. Spine 25, 331.

Diotalevi, L., Bailly, N., Wagnac, E., Mac-Thiong, J-M., Goulet, J., Petit, Y., 2020. Dynamics of spinal cord compression with different patterns of thoracolumbar burst fractures: Numerical simulations using finite element modelling. Clinical Biomechanics 72, 186194. https://doi.org/10.1016/j.clinbiomech.2019.12.023 
Foerster, O., 1936. Symptomatologie der Erkrankungen des Rückenmarks und seiner Wurzeln, in: Foerster, O., Gagel, O., Környey, St., Lotmar, F., Marburg, O., Stenvers, H.W. (Eds.), Rückenmark Hirnstamm · Kleinhirn, Handbuch der Neurologie. Springer Berlin Heidelberg, Berlin, Heidelberg, pp. 1-403. https://doi.org/10.1007/978-3-642-507762_1

Fradet, L., Arnoux, P.J., Callot, V., 2014. Finite element study of cervical spinal dislocation with spinal cord injury preliminary results. Presented at the Proceedings of SIMBIO-M, Marseille, France.

Fradet, L., Arnoux, P.-J., Callot, V., Petit, Y., 2016. Geometrical variations in white and gray matter affect the biomechanics of spinal cord injuries more than the arachnoid space. Adv. Mech. Eng. 8, 1687814016664703. https://doi.org/10.1177/1687814016664703

Gore, D.R., Sepic, S.B., Gardner, G.M., 1986. Roentgenographic findings of the cervical spine in asymptomatic people. Spine 11, 521-524. https://doi.org/10.1097/00007632198607000-00003

Jimenez, O., Marcillo, A., Levi, A.D.O., 2000. A histopathological analysis of the human cervical spinal cord in patients with acute traumatic central cord syndrome. Spinal Cord 38, 532537. https://doi.org/10.1038/sj.sc.3101052

Kim, Y.H., Khuyagbaatar, B., Kim, K., 2013. Biomechanical effects of spinal cord compression due to ossification of posterior longitudinal ligament and ligamentum flavum: a finite element analysis. Medical engineering \& physics 35, 1266-1271.

Li, X.-F., Dai, L.-Y., 2010. Acute Central Cord Syndrome: Injury Mechanisms and Stress Features. Spine 35, E955. https://doi.org/10.1097/BRS.0b013e3181c94cb8

Martin, D., Schoenen, J., Lenelle, J., Reznik, M., Moonen, G., 1992. MRI-pathological correlations in acute traumatic central cord syndrome: case report. Neuroradiology 34, 262-266. https://doi.org/10.1007/BF00588177

Mattucci, S.F., Cronin, D.S., 2015. A method to characterize average cervical spine ligament response based on raw data sets for implementation into injury biomechanics models. J. Mech. Behav. Biomed. Mater. 41, 251-260.

Mattucci, S.F., Moulton, J.A., Chandrashekar, N., Cronin, D.S., 2012. Strain rate dependent properties of younger human cervical spine ligaments. J. Mech. Behav. Biomed. Mater. $10,216-226$. 
McKinley, W., Santos, K., Meade, M., Brooke, K., 2007. Incidence and Outcomes of Spinal Cord Injury Clinical Syndromes. J. Spinal Cord Med. 30, 215-224.

Molliqaj, G., Payer, M., Schaller, K., Tessitore, E., 2014. Acute traumatic central cord syndrome: A comprehensive review. Neurochirurgie 60, 5-11. https://doi.org/10.1016/j.neuchi.2013.12.002

Morishita, Y., Maeda, T., Naito, M., Ueta, T., Shiba, K., 2013. The pincers effect on cervical spinal cord in the development of traumatic cervical spinal cord injury without major fracture or dislocation. Spinal Cord 51, 331-333. https://doi.org/10.1038/sc.2012.157

Muhle, C., Metzner, J., Weinert, D., Falliner, A., Brinkmann, G., Mehdorn, M.H., Heller, M., Resnick, D., 1998. Classification system based on kinematic MR imaging in cervical spondylitic myelopathy. Am. J. Neuroradiol. 19, 1763-1771.

Nathan, P.W., Smith, M.C., 1955. LONG DESCENDING TRACTS IN MANI. REVIEW OF PRESENT KNOWLEDGE. Brain 78, 248-303. https://doi.org/10.1093/brain/78.2.248

Nishida, N., Kato, Y., Imajo, Y., Kawano, S., Taguchi, T., 2012. Biomechanical analysis of cervical spondylotic myelopathy: The influence of dynamic factors and morphometry of the spinal cord. J. Spinal Cord Med. 35, 256-261. https://doi.org/10.1179/2045772312Y.0000000024

Ogino, H., Tada, K., Okada, K., Yonenobu, K., Yamamoto, T., Ono, K., Namiki, H., 1983. Canal diameter, anteroposterior compression ratio, and spondylotic myelopathy of the cervical spine. Spine 8, 1-15.

Panzer, M.B., Fice, J.B., Cronin, D.S., 2011. Cervical spine response in frontal crash. Med. Eng. Phys. 33, 1147-1159. https://doi.org/10.1016/j.medengphy.2011.05.004

Quencer, R.M., Bunge, R.P., Egnor, M., Green, B.A., Puckett, W., Naidich, T.P., Post, M.J.D., Norenberg, M., 1992. Acute traumatic central cord syndrome: MRI-pathological correlations. Neuroradiology 34, 85-94. https://doi.org/10.1007/BF00588148

Schmidt, H., Heuer, F., Simon, U., Kettler, A., Rohlmann, A., Claes, L., Wilke, H.-J., 2006. Application of a new calibration method for a three-dimensional finite element model of a human lumbar annulus fibrosus. Clin. Biomech. 21, 337-344.

Schneider, R.C., Cherry, G., Pantek, H., 1954. The syndrome of acute central cervical spinal cord injury; with special reference to the mechanisms involved in hyperextension injuries of cervical spine. J. Neurosurg. 11, 546-577. https://doi.org/10.3171/jns.1954.11.6.0546 
Schneider, R.C., Thompson, J.M., Bebin, J., 1958. THE SYNDROME OF ACUTE CENTRAL CERVICAL SPINAL CORD INJURY. J. Neurol. Neurosurg. Psychiatry 21, 216-227.

Shedid, D., Benzel, E.C., 2007. CERVICAL SPONDYLOSIS ANATOMYPATHOPHYSIOLOGY AND BIOMECHANICS. Neurosurgery

60 ,

S1-7-S1-13. https://doi.org/10.1227/01.NEU.0000215430.86569.C4

Shirazi-Adl, A., Ahmed, A., Shrivastava, S., 1986. A finite element study of a lumbar motion segment subjected to pure sagittal plane moments. J. Biomech. 19, 331-350.

Song, J., Mizuno, J., Inoue, T., Nakagawa, H., 2006. Clinical evaluation of traumatic central cord syndrome: emphasis on clinical significance of prevertebral hyperintensity, cord compression, and intramedullary high-signal intensity on magnetic resonance imaging. Surg. Neurol. 65, 117-123. https://doi.org/10.1016/j.surneu.2005.06.045

Stevenson, C.M., Dargan, D.P., Warnock, J., Sloan, S., Espey, R., Maguire, S., Eames, N., 2016. Traumatic central cord syndrome: neurological and functional outcome at 3 years. Spinal Cord 54, 1010-1015. https://doi.org/10.1038/sc.2016.34

Tani, T., Yamamoto, H., Kimura, J., 1999. Cervical spondylotic myelopathy in elderly people: a high incidence of conduction block at C3-4 or C4-5. J. Neurol. Neurosurg. Psychiatry 66, 456-464. https://doi.org/10.1136/jnnp.66.4.456

Taso, M., Fradet, L., Callot, V., Arnoux, P.J., 2015. Anteroposterior compression of the spinal cord leading to cervical myelopathy: a finite element analysis. Comput. Methods Biomech. Biomed. Engin. 18, 2070-2071. https://doi.org/10.1080/10255842.2015.1069625

Thompson, C., Mutch, J., Parent, S., Mac-Thiong, J.-M., 2015. The changing demographics of traumatic spinal cord injury: An 11-year study of 831 patients. J. Spinal Cord Med. 38, 214-223. https://doi.org/10.1179/2045772314Y.0000000233

Wagnac, E., Arnoux, P.-J., Garo, A., Aubin, C.-E., 2012. Finite element analysis of the influence of loading rate on a model of the full lumbar spine under dynamic loading conditions. Med. Biol. Eng. Comput. 50, 903-915.

Wagnac, E., Arnoux, P.-J., Garo, A., El-Rich, M., Aubin, C.-E., 2011. Calibration of hyperelastic material properties of the human lumbar intervertebral disc under fast dynamic compressive loads. J. Biomech. Eng. 133, 101007. https://doi.org/10.1115/1.4005224

Yu, Y.L., Stevens, J.M., Kendall, B., Boulay, G.H. du, 1983. Cord shape and measurements in cervical spondylotic myelopathy and radiculopathy. Am. J. Neuroradiol. 4, 839-842. 
508 Appendix 1: Results from the dynamic calibration of disks: Finite element model material 509 properties

511 Appendix 1a: Material properties of disk

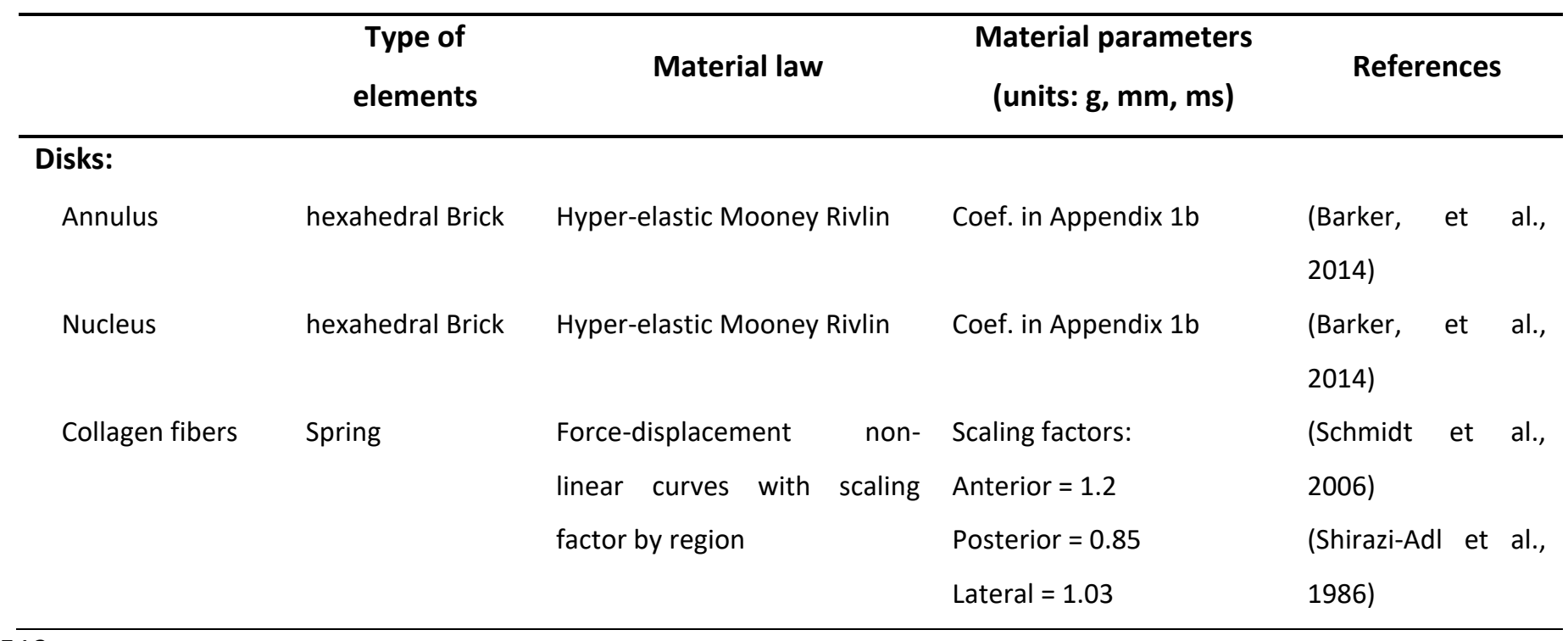


522 Appendix 1b: Coefficients of the Mooney-Rivlin hyper-elastic material laws of the annulus and 523 nucleus for each spinal functional unit

\begin{tabular}{|c|c|c|c|c|c|c|c|c|}
\hline Spinal unit & $\mathrm{C} 2-\mathrm{C} 3$ & C3-C4 & C4-C5 & C4-C5 & C5-C6 & C6-C7 & $\mathrm{C} 6-\mathrm{C7}$ & C7-T1 \\
\hline \multicolumn{9}{|l|}{ Annulus } \\
\hline \multicolumn{9}{|l|}{ Anterior } \\
\hline$C_{10}$ & 0.72 & 2.88 & 0.11 & 0.36 & 0.72 & 0.72 & 7.20 & 10.80 \\
\hline $\mathrm{C}_{01}$ & -0.18 & -0.72 & -0.03 & -0.09 & -0.18 & -0.18 & -1.80 & -2.70 \\
\hline \multicolumn{9}{|l|}{ Lateral } \\
\hline $\mathrm{C}_{10}$ & 0.72 & 2.88 & 0.11 & 0.36 & 0.72 & 0.18 & 10.80 & 10.80 \\
\hline $\mathrm{C}_{01}$ & -0.18 & -0.72 & -0.03 & -0.09 & -0.18 & -0.05 & -2.70 & -2.70 \\
\hline \multicolumn{9}{|l|}{ Posterior } \\
\hline $\mathrm{C}_{10}$ & 0.72 & 5.76 & 0.45 & 0.36 & 5.76 & 0.07 & 10.80 & 5.76 \\
\hline $\mathrm{C}_{01}$ & -0.18 & -1.44 & -0.11 & -0.09 & -1.44 & -0.02 & -2.70 & -1.44 \\
\hline \multicolumn{9}{|l|}{ Nucleus } \\
\hline $\mathrm{C}_{10}$ & 0.96 & 7.68 & 1.92 & 0.48 & 0.96 & 0.96 & 0.96 & 14.40 \\
\hline $\mathrm{C}_{01}$ & -0.72 & -5.76 & -1.44 & -0.36 & -0.72 & -0.72 & -0.72 & -10.80 \\
\hline
\end{tabular}

